\title{
Les ajustements des professionnels de la médiation au musée face aux enjeux de la culture numérique
}

The Adjusted Role of the Museum Mediator Faced with the Challenges of Digital Culture

\section{Éva Sandri}

\section{OpenEdition}

\section{Journals}

Édition électronique

URL : http://journals.openedition.org/edc/6557

DOI : $10.4000 /$ edc. 6557

ISSN : 2101-0366

\section{Éditeur}

Université de Lille

\section{Édition imprimée}

Date de publication : 1 juin 2016

Pagination : 71-86

ISBN : 978-2-917562-15-4

ISSN : $1270-6841$

\section{Référence électronique}

Éva Sandri, « Les ajustements des professionnels de la médiation au musée face aux enjeux de la culture numérique », Études de communication [En ligne], 46 | 2016, mis en ligne le 01 juin 2018, consulté le 10 décembre 2020. URL : http://journals.openedition.org/edc/6557 ; DOI : https://doi.org/ 10.4000/edc. 6557 
Les ajustements des professionnels de la médiation au musée face aux enjeux de la culture numérique

The Adjusted Role of the Museum Mediator Faced with the Challenges of Digital Culture

Éva Sandri

Université d'Avignon, Centre Norbert Élias,

Équipe Culture et Communication

eva.sandri@univ-montp3.fr 
Cet article vise à observer les ajustements du métier de médiateur au musée face au développement de dispositifs de médiation numérique. Nous interrogeons plus précisément le rôle des médiateurs dans deux musées d'ethnographie intégrant des technologies numériques et étant amenés à concevoir ou à adapter des dispositifs de médiation. Cette enquête révèle la plasticité des métiers de la médiation culturelle ainsi que des logiques d'opposition, d'accommodation et d'inventivité des médiateurs qui tentent d'adapter les dispositifs technologiques qui ne correspondent pas à leur conception de la médiation.

Mots-clés : médiation culturelle, culture numérique, musée, métiers, ajustement.
This article examines the ways in which the professional activity of the museum mediator has undergone adjustments as a result of the development of digital mediation devices. We explore the role of mediators in two ethnographic museums which have adopted digital technologies and have designed or adapted mediation devices. Our survey reveals the plasticity of cultural mediation occupations and points out the strategies of opposition, adaptation and inventiveness among museum mediators who try to adapt technological devices which do not correspond to their conception of cultural mediation.

Keywords: cultural mediation, digital humanities, museums, profession, adjustment. 
Dans un contexte marqué par une injonction à l'utilisation des dispositifs numériques dans le domaine culturel (Jeanneret, 2007 et 2014; Labelle, 2007), nous interrogeons dans cet article les logiques d'ajustement de professionnels de la médiation dans deux musées d'ethnographie ${ }^{1}$ donnant à voir des façons de concevoir et d'adapter des outils numériques dans les activités de médiation. Nous cherchons à comprendre dans quelle mesure ils sont à l'origine de ces transitions en questionnant leur degré d'autonomie. Disposent-ils d'espace pour porter un regard critique sur la façon dont se mettent en place les dispositifs numériques? Quel degré de liberté et d'expertise ont-ils lors du choix des dispositifs? À ce titre, la notion d'ajustement permet d'appréhender ce qui se passe en termes non pas de changement professionnel mais de repositionnement, ce qui évite de se focaliser exclusivement sur les éléments qui évoluent. Dans ce contexte, les modèles qui perdurent et résistent doivent être pris en compte. Nous interrogeons de cette façon la question de la stabilité et de l'inertie (Le Marec et Babou, 2003) en portant notre attention sur la dialectique entre mutations et permanence dans le cadre de ces repositionnements. II s'agit in fine d'examiner la façon dont certains professionnels de la médiation réagissent, s'adaptent et s'ajustent face aux dispositifs numériques et aux injonctions qui les accompagnent.

La réalisation d'une enquête ethnographique, constituée d'entretiens et de temps d'observation lors des activités de médiation, permet d'observer les modalités d'intégration des usages des technologies numériques dans les discours et les pratiques de différents professionnels (responsables du service de la médiation, médiateurs et guides-conférenciers) en fonction de leurs attentes et de leur conception de la médiation culturelle notamment lorsqu'ils sont impliqués dans le choix des dispositifs, voire dans la réalisation de visites.

Pour identifier plusieurs stratégies d'ajustement de la médiation en contexte d'intégration de technologies numériques, le terrain d'étude réunit deux musées qui se confrontent à ces évolutions dans des temporalités et des contextes fort différents. Le musée McCord (musée d'histoire de la ville de Montréal) présente une expérience antérieure de plus de vingt ans dans l'usage d'outils numériques, en relation avec la valorisation, sur son site web, de son fonds d'archives photographiques (Young, 2001). Ce musée accroît la visibilité de ces documents numériques dans l'exposition à travers l'acquisition de dispositifs mobiles de type iPod permettant de consulter des contenus issus des collections en ligne durant les activités de médiation (visites guidées et ateliers).

À l'inverse, le Museon Arlaten (musée d'ethnographie provençale d'Arles) est une institution plus traditionnelle. Créé à l'initiative du poète Frédéric Mistral en1899, le musée présentait à l'origine la culture provençale rurale de la fin du

1 Nous tenons à remercier le Museon Arlaten ainsi que le musée Mc Cord pour nous avoir permis de mener des entretiens et d'effectuer des observations durant les activités de médiation et les réunions. 
XVIII ${ }^{e}$ siècle. Ce musée s'est engagé depuis 2009 dans un projet de redéfinition de l'ensemble de sa muséographie en pariant sur l'apport des technologies numériques pour mettre à distance le discours folkloriste de l'époque et pour présenter dans le même temps les interrogations actuelles des musées d'ethnographie (Séréna Allier, 2008).

\section{1.}

La notion d'ajustement pour aborder les relations des professionnels de la médiation aux technologiques numériques

La question des ajustements professionnels face aux enjeux de l'usage des technologies numériques dans le domaine culturel a fait l'objet depuis une dizaine d'années d'enquêtes qui mettent en avant diverses stratégies d'adaptation de la part des professionnels (Le Marec et Babou, 2003; Rizza, 2013; Fabre, 2012). Ces repositionnements prennent généralement forme dans l'ajustement des médiateurs vis-à-vis de tâches qui leur sont demandées lors des projets numériques.

Observer ces ajustements demande tout d'abord de considérer que les usagers des technologies ne sont pas passifs face à ces dispositifs mais qu'ils peuvent faire preuve de résistance à travers des logiques inventives visant à contourner les usages attendus (De Certeau, 1980; Landowski, 2014). Les ruses, tactiques et autres arrangements que Michel de Certeau analyse sous le terme de braconnage décrivent l'existence d'une «activité silencieuse, transgressive, ironique ou poétique» $(1980,49)$ des usagers. Cependant, ces relations entre les professionnels et les dispositifs technologiques ne se résument pas à une question de choix entre adoption ou rejet mais donnent à voir des négociations plus fines menant à des logiques créatives. L'ajustement consiste en effet en des interactions qui s'affranchissent des conventions propres à un type d'activité, permettant ainsi à des sujets de s'accorder sur un référent qui leur semble juste et qui ouvre «la possibilité de dynamiques créatrices et non plus reproductrices de sens et de valeur» (Landowski, 2014; 2006). Cette stratégie d'invention relève davantage des logiques de créativité sociale et décrit l'accord des individus sur un certain nombre de décisions communes (op. cit.).

Cependant, la limite de ce modèle serait de penser ces stratégies comme de simples phénomènes subversifs décrivant des accidents et des contre-pratiques. Ainsi, pour éviter l'écueil d'une conception binaire qui décrirait les bons usages face aux résistances, nous souhaitons observer de manière plus globale les pratiques des médiateurs en relevant l'ensemble des logiques d'appropriation. Nous considérons alors que les phénomènes d'ajustement par l'opposition font pleinement partie des usages des dispositifs numériques (Cotte, 2007; Jutant, 2011). À ce titre, le concept d'ajustement théorisé par Camille Jutant 
(2011) dans le cadre d'une étude sur les pratiques de visite au musée prend en compte des logiques d'ajustement plus proches de l'adaptation que de la résistance. L'ajustement est défini par cette chercheuse comme l'évolution du comportement des visiteurs de musée lors de leur consultation des dispositifs de l'exposition (textes, multimédias) en fonction de leur culture muséale. Dans ce contexte, l'ajustement permet de décrire «l'adéquation plus ou moins grande à une succession de tâches" $(2011,222)$ et de rendre compte de la négociation du visiteur avec l'expérience que le dispositif lui propose. Cette définition de l'ajustement plus proche de l'appropriation que de la résistance met l'accent sur la façon dont l'usager fait appel à sa culture du musée et des technologies pour se positionner face à un dispositif à travers un jugement critique.

Ainsi, l'activité consistant à exprimer une opinion à propos des dispositifs numériques donne aux usagers l'opportunité de repenser leurs pratiques. Ils peuvent alors proposer un regard réflexif sur les dispositifs menant à un "ajustement méfiant» (Jeanneret, 2008, 126²) favorisant les stratégies d'adaptation. Cette attitude leur permet de s'ajuster afin de «faire avec les dispositifs et les gestes de médiation institués» (Jeanneret, 2014, 87) dans un contexte d'injonction au numérique.

Nous avons utilisé ce concept d'ajustement pour analyser de quelles manières les professionnels de la médiation s'impliquent dans la culture numérique, assument de nouvelles fonctions, décrivent et utilisent des outils relevant des technologies numériques. Ci-dessous les ajustements observés dans les deux terrains de recherche sont présentés en deux temps. Nous cherchons à comprendre d'abord le point de vue des professionnels face à l'utilisation des technologies dans les activités de médiation. Nous décrivons ensuite leurs pratiques professionnelles dans des contextes d'actualisation d'activités de médiation, d'acquisition de dispositifs et d'utilisation des dispositifs avec le public.

\section{2. \\ L'exercice d'un jugement face à la réquisition numérique}

Dans les deux terrains, nous relevons une situation que l'on peut qualifier de réquisition numérique. La réquisition est un phénomène de prescription qui encourage fortement des pratiques tout en opérant une partition sociale liée au respect de ces pratiques. Elle représente "l'ensemble des moyens techniques, idéologiques, formels, qui contribuent à pousser toute une société à

2 Yves Jeanneret fait ici référence aux travaux de Louis Quéré (1982) et de Richard Hoggart (1957). Tous deux ont étudié la capacité des individus à convoquer leurs propres connaissances pour porter un regard à la fois réflexif et critique sur leurs pratiques médiatiques. 
adopter des outils médiatiques sans nécessairement avoir de raison ou de but particulier pour y avoir recours et qui tend à marginaliser ceux qui n'y ont pas recours» (Jeanneret, 2014, 14). II s'agit d'un «appel à l'activité» (ibid., 120) dans un cadre normatif, donnant à voir une orthopraxie (Labelle, 2007). La notion d'ajustement comprise comme une capacité de jugement amène à observer la manière dont les professionnels de la médiation construisent leur point de vue au sein de ce contexte injonctif.

\subsection{Ni technolophiles...}

Les enquêtés des deux musées critiquent globalement une réception trop enthousiaste des dispositifs technologiques, caractérisée par un regard plus centré sur la technologie que sur les objectifs à long terme pour la médiation. Ils disent se sentir acculés dans des logiques qui les obligent à acquérir des technologies récentes alors même que certains dispositifs déjà connus permettent d'atteindre les mêmes objectifs. Une perception trop enthousiaste de ces dispositifs nuit selon le responsable du service des publics du Museon Arlaten à une réflexion sur leur pertinence:

× «Il y a un effet de mode, je me suis aperçue en rencontrant des gens qu'il n'y avait pas de compréhension de ce qu'était vraiment la réalité augmentée», Responsable du service des publics.

Il évoque également certaines représentations sociales concernant la médiation numérique, notamment la tendance à ramener la réflexion aux seuls supports, sans penser leur nécessité. II reproche au contexte actuel de chercher l'innovation dans des supports technologiques et non dans des activités de médiation globales, par exemple en se focalisant sur la réalité augmentée et non sur son inscription dans un scénario d'activité. L'intégration de dispositifs numériques est également critiquée par les animateurs du musée McCord qui ont l'impression que le musée impose au nom de l'innovation un outil redondant au sein d'activités de médiation dont ils sont satisfaits. Selon eux, les outils numériques semblent alors rajoutés dans l'offre de médiation sans qu'il y ait eu au préalable une discussion commune sur les besoins véritables des publics et des médiateurs. Certains propos donnent à voir une frustration vis-à-vis de cette modification de l'organisation des visites:

× «Les animateurs ils sont partagés, parce qu'ils voient que le numérique est plus un ajout qu'une nécessité. On a un contenu, l'animateur pouvait le faire sans support numérique, mais on se tape le numérique parce qu'il y a une subvention ", Coordinateur de l'action éducative.

L'enquêté dénonce dans cet extrait l'acquisition non nécessaire d'un dispositif numérique (ici un iPod) qui n’apporte pas d'élément supplémentaire aux visiteurs comme aux animateurs lors de l'activité de médiation. On peut également se demander dans ce contexte quels sont les espaces que les professionnels peuvent investir pour présenter leurs initiatives de médiation 
non numériques. Dans quelle mesure les médiateurs ont-ils l'occasion de faire part de leurs doutes à propos de la pertinence de ces dispositifs? A ce sujet, si les enquêtés font part de leurs craintes durant les entretiens ainsi que lors des réunions de l'équipe de médiation auxquelles nous avons pu assister, ils indiquent cependant avoir plus de difficultés à émettre ces opinions lors des réunions qui rassemblent toutes les équipes du musée.

\subsection{Ni technophobes}

Les responsables du service de médiation des deux musées indiquent acquérir par eux-mêmes des connaissances sur les pratiques numériques via les réseaux sociaux, la veille documentaire et les journées d'études. L'intérêt de ces professionnels pour le lien avec le public en ligne les encourage à mettre en place un travail de gestionnaire de communauté, sans formation officielle, mais en suivant les pratiques d'autres gestionnaires influents. À ce titre, la communauté muséogeek ${ }^{3}$, le wiki muzeonum ${ }^{4}$ et le dispositif museomix ${ }^{5}$ ont favorisé cet apprentissage. Ainsi, le métier de médiateur tend à inclure les savoir-faire de la médiation numérique, donnant à voir une formation en autodidacte menant les médiateurs interrogés à assurer des fonctions de médiation numérique alors même que l'institution n'a pas spécifiquement créé de poste dédié. Dans ce contexte, les médiateurs deviennent alors des éléments moteurs du développement de la médiation numérique et expliquent avoir un rôle de passeur de la culture numérique au sein de leur propre équipe avec une volonté de former leurs collègues. On note par exemple un fort intérêt du responsable du service des publics du Museon Arlaten pour les médias sociaux, ainsi qu'une volonté d'engager les autres équipes du musée dans ce travail d'interaction avec les publics:

× «Je suis très intéressée par le public virtuel, le community management, au final il n'y a que moi qui le fait», "J'aimerais une petite conférence où on peut poser toutes les questions via Twitter avec les conservateurs de musée, j'ai beaucoup d'idées et j'épuise un peu tout le monde et ça fait peur à tout le monde... Mais j'aimerais les impliquer!», Responsable du service des publics.

Ce souhait de connaître les pratiques des usagers des dispositifs numériques a été relevé dans les discours des médiateurs des deux musées. Pour les enquêtés, une formation à la médiation par le numérique leur permettrait de mieux comprendre les enjeux actuels des musées (notamment ceux qui

3 Le terme museogeek fait référence à quatre communautés de professionnels des musées et du numérique. Ces communautés sont décrites sur leur site internet: <http://museogeeks.com/>.

4 Le wiki mis en place sur le site de muzeonum rassemble les ressources relatives à l'utilisation des technologies dans les musées. Il est animé par la communauté museogeek: <http://www.muzeonum.org/wiki/doku.php>.

5 Site internet de l'évènement museomix: < http://www.museomix.org/>. 
expérimentent des stratégies numériques) afin de faire des choix éclairés à ce sujet et in fine de s'approprier les dispositifs à acquérir. Leur curiosité envers les usages numériques se double donc d'un regard critique vis-à-vis des discours promotionnels accompagnant les technologies.

\section{3. \\ La réflexivité: une ressource pour la négociation et l'adaptation}

En émettant un jugement critique à propos des dispositifs numériques et en confrontant ces jugements avec ce qu'ils anticipent des réactions des visiteurs, les professionnels se positionnent dans une logique d'appropriation des dispositifs visant à les orienter vers leurs objectifs en termes de médiation. Ainsi, c'est la capacité des acteurs à porter un regard réflexif sur leurs pratiques qui leur sert de ressource pour ajuster les dispositifs à leur conception de la médiation.

À ce titre, si dans les deux musées nous remarquons que les médiateurs décrivent leurs pratiques numériques en y apportant un important recul critique, il faut distinguer le cas du musée McCord où les ajustements servent à actualiser les dispositifs numériques existants du Museon Arlaten où l'objectif est d'acquérir de nouveaux dispositifs servant une stratégie numérique globale.

Au musée McCord, nous avons observé la réflexivité des acteurs à propos de leurs pratiques concernant des dispositifs précis. Cette même réflexivité est à l'œuvre au Museon Arlaten et s'inscrit de surcroît dans un projet de rénovation basé sur le concept de distance critique, ce qui encourage les professionnels à fréquemment interroger leurs relations aux dispositifs numériques et à anticiper davantage les réactions des futurs visiteurs. Ainsi, le Museon Arlaten s'empare de la réflexivité à un niveau institutionnel puisque la mise à distance de l'ancienne muséographie par les dispositifs numériques fait pleinement partie du projet de rénovation. Cette situation donne à voir chez les acteurs un discours réflexif approfondi qui leur permet de s'adapter davantage aux dispositifs.

\subsection{L'ajustement par actualisation des dispositifs}

Nous prendrons l'exemple de deux activités du musée McCord pour lesquelles on observe des logiques de conception et d'ajustement différentes: I'atelier I-Montréal- Le collectionneur et la visite du Golden Square Mile. 


\section{I-Montréal-Le collectionneur: une logique d'invention}

Pour développer cette activité ${ }^{6}$, les médiateurs utilisent un dispositif technologique déjà existant au musée (un iPod servant traditionnellement d'audioguide). Il y a quelques années, le service des technologies du musée avait commandé un lot d'iPod destiné à la consultation des bases de données du musée lors des visites, mais sans qu'il y ait une réflexion au préalable avec le service des publics à propos d'une future activité de médiation. A partir de ce dispositif existant, le service des publics a ensuite imaginé l'activité de visite I-Montréal - Le Collectionneur. Le service multimédia du musée explique d'ailleurs que c'est grâce aux activités pensées par le service des publics que I'utilisation des fonctionnalités de l'iPod a pris davantage de sens. Cet exemple montre qu'il n'y a pas d'intégration soudaine et radicale des technologies numériques dans les activités de médiation mais plutôt des logiques qui visent à donner une seconde fonction à un support déjà existant.

Ce type d'ajustement visant à étendre les possibilités d'utilisation d'un support numérique donne à voir une logique d'ajustement reposant sur l'invention et témoigne d'un accord des médiateurs sur certaines pratiques communes rendant possible la flexibilité et la créativité sémiotique pointée par Landowski (ibid., 2014).

\section{Golden Square Mile: entre accommodation et détournement}

Le dispositif Golden Square Mile donne à voir une logique d'ajustement différente. Le service des publics du musée s'est basé sur une activité déjà existante, consistant en une visite d'un quartier de la ville s'appuyant sur des photographies d'archives. Cette activité a ensuite été modifiée en remplaçant les documents imprimés par l'utilisation de ce même iPod. L'activité se compose d'une visite d'un quartier du centre historique de Montréal situé près du musée McCord. II s'agit d'une visite guidée classique comportant des arrêts devant des bâtiments remarquables et dans laquelle les visiteurs sont équipés d'iPod

6 L'objectif de l'activité I-Montréal-Le collectionneur est de faire comprendre à un public scolaire les étapes d'un processus de collecte d'objets menant à la constitution d'une collection puis d'une exposition. Dans cette activité, les enfants sont invités à sélectionner les photographies de plusieurs objets de l'exposition afin de constituer leur propre collection sur un thème donné (par exemple: la mode, l'alimentation). Ils choisissent les photographies numériques de ces objets sur un iPod prêté par le musée qui propose un accès à la base de données de la collection permanente. Ensuite ils impriment ces photographies et créent une maquette en carton de leur exposition idéale où ils placent les photographies imprimées de ces objets. Dans cette activité, ce ne sont pas tant pas les objets et les savoirs qui les entourent qui sont au centre des objectifs mais bien le fait de susciter chez les élèves un questionnement sur la façon de choisir des objets dans une collection thématique et d'effectuer des choix scénographiques. Notons également qu'il ne s'agit pas d'un dispositif strictement numérique mais d'une activité complexe constituée d'alternances entre l'utilisation de supports technologiques (iPod) et de supports imprimés (cartons, papiers pour la réalisation de la maquette). 
leur donnant accès à l'application Musée Urbain MTL, contenant des informations contextuelles sur les monuments. Chaque arrêt devant un bâtiment est également l'occasion pour le guide-conférencier de raconter l'évolution du paysage urbain depuis le XIX ${ }^{e}$ siècle. En parallèle, les visiteurs doivent se repérer sur le plan du quartier disponible sur l'application et sélectionner le bâtiment dont parle le guide. Ils ont ainsi accès à des photographies du fonds Notman appartenant au musée, représentant des photos de ces mêmes bâtiments à une époque antérieure. Pour cette visite, le musée McCord utilise l'application Musée Urbain MTL qui avait été initialement réalisée pour des visites autonomes de la ville. Cette application a recours à la réalité augmentée pour superposer sur les bâtiments récents les images du passé. Avant l'utilisation de l'application, les guides utilisaient des photographies imprimées pour montrer au public les images d'archives du fonds Notman et faisaient faire du dessin d'observation devant les bâtiments ainsi que des ateliers de collage.

La création de l'application a alors été une façon de modifier la visite traditionnelle en ajoutant la manipulation d'un dispositif technologique, même si les activités manuelles ont perduré. Ainsi, contrairement à l'activité I-Montréal- Le Collectionneur, on observe ici une activité déjà existante qui se trouve actualisée par un support numérique tout en conservant des éléments traditionnels. Concernant l'application Musée Urbain MTL, on remarque une réutilisation d'une application existante dédiée à la visite autonome dans un autre contexte de médiation: la visite guidée.

Cependant, le médiateur qui explique la genèse de ce projet indique qu'il n'approuve pas totalement la pertinence du dispositif. Selon lui, il s'agirait pour le musée de justifier l'acquisition des iPod sans que l'évolution de cette activité ne se fasse dans l'objectif de répondre à un besoin précis des visiteurs. On observe donc une pression à l'ajustement des activités visant à légitimer les dépenses faites par l'institution dans le domaine du numérique et non une réponse à une demande des médiateurs.

Cette activité de médiation est néanmoins décrite comme étant flexible pour les guides-conférenciers. Le coordinateur du service des publics affirme qu'il accepte de modifier le scénario de l'activité pour pouvoir adapter la visite au profil de chaque animateur. II travaille en effet avec des médiateurs plus ou moins impliqués dans la médiation numérique. Il indique notamment avoir approuvé la décision d'un guide qui, craignant un manque de compétences du public lors de la manipulation de l'iPod, n'a pas souhaité proposer cet outil (pourtant indiqué dans la description de l'activité) à un groupe de sexagénaires lors d'une visite. Le responsable du service explique tolérer ce type de refus, même si le choix de ce guide ayant préféré conserver le support papier plutôt que d'utiliser l'iPod diffère des choix du musée en termes de politique numérique et d'image institutionnelle. C'est donc à partir de leur regard réflexif sur les formes actuelles de médiation et de leur anticipation des réactions des publics que les médiateurs ajustent l'utilisation des dispositifs dans un compromis 
entre la volonté de l'institution et leurs aspirations en termes de médiation.

À travers ces deux exemples nous observons que l'introduction de dispositifs numériques donne moins à voir une logique linéaire (passant systématiquement par la commande d'un matériel associé à un usage précis) qu'une adaptation de chacun des médiateurs vis-à-vis d'un dispositif existant"

\subsection{L'ajustement lors de l'acquisition de dispositifs}

Observer la longue période de rénovation du Museon Arlaten nous a donné l'occasion de saisir des enjeux que nous n'avions pas pu appréhender au musée McCord tels que les débats réalisés en amont lors de l'acquisition de dispositifs numériques pour la médiation. En assistant à des réunions à divers moments du projet (rédaction des marchés publics et des cahiers des charges), nous avons décelé trois situations d'ajustements: des ajustements par rapport aux prestataires, des ajustements entre les différentes équipes du musée et enfin des auto-ajustements chez les médiateurs. Nous aborderons dans cette partie les stratégies des médiateurs lors de leurs interactions avec les autres services du musée tels que le chargé de mission multimédia (responsable de l'expertise technologique) et l'équipe recherche et muséographie (en charge des contenus éditoriaux des dispositifs).

\section{Les ajustements du musée par rapport aux prestataires}

Si la gestion de projet de conception de dispositif de médiation suit généralement un ordre allant de la définition des besoins de l'usager vers le choix des contenus puis du dispositif technique permettant sa réalisation, la collaboration du Museon Arlaten avec la maitrise d'œuvre (les architectes) révèle des logiques de travail qui s'écartent de ce modèle. En effet, les architectes ayant pour mission l'achat du mobilier et le musée ayant la responsabilité du projet scientifique et culturel, la recherche de cohérence entre ces deux dimensions occasionne de nombreux débats et compromis. Cet arbitrage peut se révéler délicat lorsque les deux parties ont des attentes divergentes vis-à-vis du nouveau musée.

À ce sujet, nous avons observé lors des entretiens des logiques de conception différentes en fonction des dispositifs. Le chargé de mission multimédia

7 Considérant qu'il y a un «biais théorique à analyser uniquement ce qui change en présupposant que c'est là seulement que se situe l'action sociale» (Le Marec et Babou, 2003), citons aussi les activités de médiation du musée McCord qui n'ont pas évolué avec les technologies numériques. Pour cela, nous avons relevé les intitulés de toutes les autres activités proposées au musée McCord: l'atelier de crochet Parent poupon, les lectures de L'heure du conte, I'activité Danses et percussions autochtones, et enfin la visite Au rythme des aînés. Ces activités n'impliquent pas ou peu l'utilisation de dispositifs numériques, ce qui montre que les logiques d'ajustement précédemment décrites ne sont pas majoritaires et n'imposent pas une norme dans l'institution. 
compare à ce titre deux logiques dans la gestion de projet à travers ce qu'il nomme le travail «à l'endroit » et celui «à l'envers». Lorsque l'équipe du musée a l'opportunité de penser en amont le contenu avant de réfléchir au dispositif numérique, la logique de conception suit un cheminement logique puisque les intentions éditoriales préexistent aux technologies. Les futurs cartels numériques ont été réalisés en suivant cet ordre:

× «On a réfléchi à l'ergonomie, au comportement dynamique du dispositif, ça c'est un travail dans le bon sens, ça c'est ce qu'on devrait faire partout», Chargé de mission multimédia.

À l'inverse, la conception des tables tactiles suit un parcours contraire puisque ce sont les architectes qui ont d'abord déterminé le matériel puis laissé au musée le soin de choisir les documents à intégrer. Les professionnels se trouvent donc face au défi de réaliser des contenus sur un support technologique imposé. Face à cette logique de travail "à l'envers», l'équipe de médiation tente d'adapter les contenus à la spécificité de la table tactile à travers des stratégies d'ajustement créatives visant à orienter les usages du dispositif.

\section{Les ajustements entre les différentes équipes du musée}

La gestion de la quantité de contenus sur les dispositifs numériques occasionne des débats autant entre le musée et les architectes qu'entre les différentes équipes du musée. Face à la tentation de proposer un très grand nombre de contenus sur les supports numériques, les différentes équipes sont attentives au risque de noyer le visiteur sous un trop plein d'informations ou sous de trop grandes stimulations audiovisuelles. Ainsi, les débats sur la surcharge informationnelle concernent surtout le service des publics et l'équipe recherche et muséographie (chargée du choix des contenus multimédias du musée). Le responsable du service des publics soulève la tendance de l'équipe recherche et muséographie à proposer un nombre important de contenus et cherche de son côté à rendre accessibles ces informations en pensant des scénarios spécifiques pour les publics néophytes:

× «Le futur musée, intellectuellement, il est super dur [...] ça réclame des connaissances et un background culturel assez important, et je trouve que c'est un musée qui ne sera pas facile quoi et donc euh je suis toujours à essayer d'apporter la voix d'un public élargi qui n'a pas fait bac plus sept en archéologie», Responsable du service des publics.

L'exemple ci-dessus donne à voir une logique d'ajustement de la part de la responsable du service des publics qui cherche à simplifier et scénariser spécifiquement les contenus choisis par l'équipe recherche et muséographie.

\section{L'auto-ajustement des médiateurs}

On remarque enfin que les médiateurs eux-mêmes indiquent qu'ils souhaitent modérer certaines de leurs ambitions lors de la conception de dispositifs 
numériques. Le responsable du service des publics décrit une négociation entre deux tendances: l'exaltation face à un imaginaire infini des dispositifs numériques (à travers l'exemple de la numérisation des collections) et la lucidité face à la réalité de la rénovation qui demande de mettre à disposition des publics une quantité de contenus raisonnable et scénarisée:

× «ll y a tellement de documents [...] je pense qu'on va se jeter sur le numérique comme la possibilité de mettre des tonnes de stockage: d'images de textes, de lettres de photos de patins et couffins, et que ça risque d'être un peu aride [...] faudra qu'on se coupe un peu les ailes en termes de contenus, on mettra moins que ce qu'on avait imaginé au départ», Responsable du service des publics.

Cet extrait témoigne d'une stratégie d'auto-ajustement par négociation puisque le responsable du service des publics cherche à contenir son envie première de proposer un nombre important de contenus dès lors qu'il imagine le comportement des futurs visiteurs. Cela permet également d'observer le regard réflexif qu'il porte sur les usages des dispositifs à travers un discours allant de l'enthousiasme à l'anticipation de la réaction des publics. Cet extrait reflète enfin les caractéristiques des dispositifs numériques attendus par les médiateurs du Museon Arlaten tels qu'ils sont décrits lors des entretiens: raisonnés, modérés, pertinents et discrets.

Ce discours des enquêtés à propos d'un numérique raisonné devient alors le symptôme d'une exigence complexe visant à trouver un équilibre entre l'aspect attractif d'une technologique numérique récente et son aspect potentiellement trop innovant qui la décrédibilise, la marquant définitivement du sceau du gadget ou de la technologie comme fin en soi. On remarque également que les enquêtés cherchent à atténuer la visibilité matérielle des dispositifs pour mettre en avant les contenus plus que le contenant. Bien qu'il soit complexe de différencier clairement ce qui relève du support technologique de ce qui relève du contenu informationnel, le discours des médiateurs donne à voir une ambivalence entre ces deux aspects du numérique (attractif d'une part et inutilement gadget d'autre part) répartis dans la dualité support/contenu.

Ainsi, afin de se positionner dans cette ambivalence, les médiateurs tentent de mettre en valeur la compatibilité entre l'innovation technologique et le sérieux scientifique de l'institution muséale (comme si l'inscription du discours du musée sur un support numérique menaçait intrinsèquement la fiabilité des informations présentées). Cette réflexion sur le degré de visibilité des dispositifs de médiation numérique, dans un contexte où l'aspect symbolique des univers muséal et technologique cristallise de nombreux stéréotypes, occasionne chez les médiateurs de nombreux questionnements à propos de l'image institutionnelle du musée.

En conclusion, nous observons dans les deux musées des logiques "d'ajustement méfiant» (Jeanneret, 2008) proches de l'appropriation, de la 
part des enquêtés, vis-à-vis de certaines manières d'intégrer des technologies numériques dans la médiation. Ces ajustements sont visibles autant dans leurs discours (à travers les critiques) que dans leurs actions (refus d'utiliser un dispositif technologique).

Cependant, la nature et la force de ces ajustements dépendent de l'ordre dans lequel sont effectués les dispositifs numériques. Nous avons en effet observé la présence de deux types de logiques de conception: lorsque les intentions éditoriales préexistent à la technologie et lorsque les médiateurs doivent s'adapter à une technologie imposée. Les ajustements sont davantage prononcés lorsque la technologie est pensée en premier, puisque les médiateurs cherchent à orienter le dispositif technologique mis à disposition en imaginant une activité qui fasse sens avec les contenus du musée. C'est le cas de la table multitouch du Museon Arlaten et de l'activité Golden Square Mile au musée McCord. Cependant, nous avons également observé des exemples d'ajustement dans des situations où les objectifs du dispositif ont été conçus avant la technologie. C'est le cas des cartels numériques du Museon Arlaten et l'activité I-Montréal - Le collectionneur au musée McCord. Dans ces cas, les médiateurs ont recours à la fois à leur regard réflexif et à leur culture de la médiation pour éprouver la malléabilité de ces dispositifs et éventuellement les transformer en les adaptant à la spécificité de leurs publics.

Enfin, par rapport au concept de réquisition numérique (Labelle, 2007; Jeanneret, 2014), nous remarquons que les professionnels des deux musées négocient avec les discours d'injonction du passage au numérique pour imposer un modèle modéré et ainsi légitimer leurs ajustements visant à utiliser les technologies de façon raisonnée. Les critiques des enquêtés à propos des dispositifs numérique existant dénoncent fréquemment ces logiques d'injonction au numérique en ayant recours à trois types d'arguments: le manque de pertinence des dispositifs, le manque de recul critique à propos des technologies actuelles et l'injonction à l'innovation. Nous notons enfin qu'ils expriment avoir des difficultés à trouver des espaces pour critiquer les logiques de mise en place des dispositifs. Ils indiquent parfois craindre de passer pour réactionnaires lorsqu'ils émettent des réserves, alors même qu'ils critiquent moins la place du numérique dans la médiation que le recours systématique à ces dispositifs.

Les espaces d'engagement et de négociation face à la culture numérique révélés par cette enquête exploratoire rendent compte de la plasticité des métiers de la médiation culturelle ainsi que des logiques d'accommodation, d'appropriation et d'inventivité des médiateurs qui tentent d'adapter les dispositifs technologiques à leur conception de la médiation. 
Bibliographie

Cotte D. (2007). «Espace de travail et logique documentaire». In Études de communication, $n^{\circ} 30$, pp.46-71.

De Certeau M. (1980). L'invention du quotidien. Vol.1: Arts de faire, Paris, Gallimard.

Fabrel. (2012). «Médiation documentaire et culturelle dans le musée». In Communications\&langages, $n^{\circ} 173$, pp.83-99.

Hoggart R. (1957). La culture du pauvre: étude sur le style de vie des classes populaires en Angleterre, Paris, Minuit.

JeanneretY. (2008). Penser la trivialité: La vie triviale des êtres culturels, Paris, Hermès Science, Lavoisier.

Jeanneret Y. (2014). Critique de la trivialité, Paris, Non Standard.

Jutant C. (2011). S'ajuster, interpréter et qualifier une pratique culturelle: Approche communicationnelle de la visite muséale, thèse de doctorat en sciences de l'information et de la communication, université d'Avignon et des Pays de Vaucluse.

LabelleS. (2007). La ville inscrite dans "la société de l'information": formes d'investissement d'un objet symbolique, thèse de doctorat en sciences de l'information et de la communication, Celsa, université Paris-Sorbonne.
LandowskiÉ. (2006). Les interactions risquées, Limoges, PUL.

Landowski É. (2014). «Accord, justesse, ajustement». In Actes

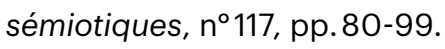

Le Marec J. et Baboul. (2003). «De l'étude des usages à une théorie des 'composites': objets, relations et normes en bibliothèque". In Jeanneret Y.(dir.), Lire, écrire, récrire. Objets, signes et pratiques des médias informatisés, Paris, BPI, pp.233-299.

QuéréL. (1982). Des miroirs équivoques: aux origines de la communication moderne, Paris, Aubier-Montaigne.

Rizza M. (2013). «La numérisation du dossier d'œuvre: Enjeux organisationnels pour les musées». In Culture\&Musées, $n^{\circ} 22$, pp.25-46.

Séréna-Allier D. (2008). «Réinventer le propos du Museon Arlaten: Une mise en abyme du musée ethnographique». In Rolland A. (dir.), De nouveaux modèles de musées? Formes et enjeux des créations et rénovations de musées en Europe: XIX ${ }^{e}-X X{ }^{e}$ siècle, Paris, L'Harmattan, pp. 213-218.

Young B. (2001). Le McCord. L'Histoire d'un musée universitaire, 1921-1996, Montréal, Hurtubise NMH. 
